\title{
Václav Chvátal
}

Some relations among invariants of graphs

Czechoslovak Mathematical Journal, Vol. 21 (1971), No. 3, 366-368

Persistent URL: http://dml.cz/dmlcz/101034

\section{Terms of use:}

(C) Institute of Mathematics AS CR, 1971

Institute of Mathematics of the Czech Academy of Sciences provides access to digitized documents strictly for personal use. Each copy of any part of this document must contain these Terms of use.

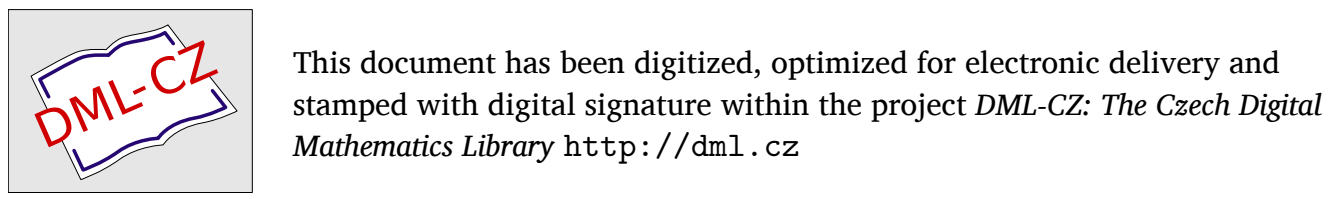




\title{
SOME RELATIONS AMONG INVARIANTS OF GRAPHS
}

\author{
Václav ChVátal, Waterloo
}

(Received January 15, 1970)

\section{INTRODUCTION}

R. E. NeTtLEton [2] investigated relations among number of vertices, connectivity, diameter, degrees of vertices and chromatic number of graphs. We shall use or improve his results to present a complete survey of the best possible bounds for the last two invariants in terms of the first three.

Our notation follows HARARY [1]. Particularly, we reserve: a letter $p$ for a number of points, $x$ for connectivity, $d$ for diameter, $\chi$ for chromatic number of a graph and $d(v)$ for a degree of a point $v$.

\section{RESULTS}

Nettleton has proved among others the following theorems:

N. 1. $\chi \geqq p /(p-\chi)$.

N. 2. If for any positive integer $j, d \geqq 2 j+1$, then $\chi \leqq p-2(x j-\varkappa+1)$.

N. 3. If $d=j+1$ for $j \geqq 3$, no point of $G$ can have degree $>p-x(j-3)-3$.

N. $4 . d-4 \leqq(p-x-3) / x$.

Later on, M. E. WATKINS [3] improved N. 4. into the following best possible form: $p \geqq x(d-1)+2$.

We shall prove two theorems:

\section{Theorem 1.}

$$
\begin{array}{ll}
p /(p-\chi) \leqq \chi \leqq p-1 \quad \text { if } \quad d=2 \\
2 \leqq \chi \leqq p-\chi(d-3)-2 \text { if } 3 \leqq d<\infty
\end{array}
$$

and these bounds are the best possible. 
Theorem 2. Given any point $v$ one has

$$
\begin{aligned}
& \varkappa \leqq d(v) \leqq p-1 \quad \text { if } d=2 \text {, } \\
& x \leqq d(v) \leqq p-2 \quad \text { if } d=3 \text {, } \\
& \varkappa \leqq d(v) \leqq p-\varkappa(d-4)-3 \text { if } 4 \leqq d<\infty
\end{aligned}
$$

and these bounds are the best possible.

These theorems show that N. 1. and N. 3. are best possible while N. 2. is not.

\section{SOME SPECIAL GRAPHS}

In this paragraph, we shall construct graphs employed as examples in proofs.

1. $G_{1}(p, x)$ where $x \leqq p-2$ is a complete $(a+1)$-partite graph $K(p-x$, $p-\varkappa, \ldots, p-\varkappa, b)$ where $p=a(p-\varkappa)+b, 0 \leqq b<p-\varkappa$. It is easy to see that $G_{1}$ is $x$-connected, its diameter is 2 and its chromatic number $a$ (if $b=0$ ), resp. $a+1$ (if $b>0$ ). In both cases, the last quantity is the least integer $\geqq p /(p-x)$.

2. $G_{2}(p, x)$ where $x \leqq p-2$ has a set of points $V_{1} \cup V_{2} \cup\{w\} V_{1}, V_{2},\{w\}$ are pairwise disjoint, $\left|V_{1}\right|=p-\varkappa-1,\left|V_{2}\right|=\varkappa$. A couple $u v$ is not a line of $G_{2}$ iff $u \in V_{1}, v=w . G_{2}$ is $x$-connected, its diameter is 2 and its chromatic number is $p-1$.

3. $G_{3}(p, \varkappa, d)$ where $d \geqq 3, \varkappa(d-1) \leqq p-2$ has a set of points $V=V_{0} \cup V_{1} \cup \ldots$ $\ldots \cup V_{d}$ where $\left|V_{0}\right|=\left|V_{d}\right|=1,\left|V_{1}\right|=p-\varkappa(d-2)-2,\left|V_{i}\right|=\varkappa$ for $i=2,3, \ldots$ $\ldots, d-1, V_{i} \cap V_{j}=\emptyset$ for $i \neq j$. A couple $u v$ is a line of $G_{3}$ iff $u \in V_{i}, b \in V_{j},|i-j|=$ $=1 . G_{3}$ is $\chi$-connected, has a diameter $d$ and a chromatic number 2 .

4. $G_{4}(p, x, d)$ where $d \geqq 3, \chi(d-1) \leqq p-2$ has the same set of points as $G_{3}$. A couple $u v$ is a line of $G_{4}$ iff $u \in V_{j}, u \in V_{j},|u-v| \leqq 1 . G_{4}$ is $x$-connected, has a diameter $d$ and a chromatic number $p-x(d-3)-2$.

\section{PROOFS}

Proof of Theorem 1: The left side of (1a) is Nettleton's result N. 1., the right side of (1a) and the left side of (1b) are trivial. Now, let $G$ be a graph with set of points $V$ and a diameter $d \geqq 3$. There are $u, v \in V$ such that $d(u, v)=d$. Put $V_{i}=\{w \in V$; $d(u, w)=i\}$ for $i=0,1, \ldots, d$. A graph $G-V_{i}$ is disconnected whenever $1 \leqq i<$ $<d$. Hence, $\left|V_{i}\right| \geqq \varkappa$ and one can find $\left\{v_{i}^{1}, v_{i}^{2}, \ldots, v_{i}^{\kappa}\right\} \subset V_{i}(1 \leqq i \leqq d)$. Write $v_{0}^{1}$ for $u$ and $v_{d}^{1}$ for $v$. Remark that $x y$ is not a line if $x \in V_{i}, y \in V_{j},|i-j| \geqq 2$ and define 
color classes $O_{1}, O_{2}, \ldots, O_{\varkappa}, E_{1}, E_{2}, \ldots, E_{\varkappa}$ by

$$
O_{j}=\left\{v_{i}^{j} ; i \text { odd }\right\}, E_{j}=\left\{v_{i}^{j} ; i \text { even }\right\} .
$$

Consider each of the remaining $p-(\varkappa(d-1)+2)$ vertices as a color class consisting of a single point. Altogether, $2 x+p-(x(d-1)+2)=p-x(d-3)+2$ color classes were introduced and $(1 \mathrm{~b})$ is proved.

To show that these bounds are best possible remember the result of Watkins and use the graphs $G_{1}(p, x), G_{2}(p, x), G_{3}(p, x, d)$ and $G_{4}(p, x, d)$ as examples.

Proof of Theorem 2: The left sides are trivial (cf. [1], Theorem 5.1) and so is the right side of (2a). The right side of $(2 \mathrm{~b})$ follows by the fact that $d(v)=p-1$ implies $d \leqq 2$. The right side of $(2 \mathrm{c})$ is Nettleton's result $\mathrm{N}$. 3 .

To see that the bounds are best possible consider graphs $G_{2}(p, \varkappa)$ and $G_{4}(p, \varkappa, d)$.

\section{References}

[1] F. Harary: Graph Theory, Addison-Wesley, Reading, Mass. 1969.

[2] R. E. Nettleton: Some Generalized Theorems on Connectivity, Canad. J. Math. XII (1960), 546-554.

[3] M. E. Watkins: A Lower Bound for the Number of Vertices of a Graph, Amer. Math. Monthly 74 (1967), 297.

Author's address: University of Waterloo, Waterloo, Ontario, Canada. 